\title{
FLUXO DIFUSIVO DE MICRONUTRIENTES CATIÔNICOS AFETADO PELO TIPO, DOSE E ÉPOCA DE INCORPORAÇÃO DE ADUBOS VERDES AO SOLO ${ }^{(1)}$
}

\author{
Rodinei Facco Pegoraro ${ }^{(2)}$, Ivo Ribeiro Silva ${ }^{(3)}$, Roberto Ferreira Novais $^{(3,5)}$, \\ Eduardo de Sá Mendonça ${ }^{(3,5)}$, Víctor Hugo Alvarez V. ${ }^{(3,5)}$, Flancer Novais \\ Nunes $^{(4)} \&$ Fabrício Oliveira Gebrim ${ }^{(4)}$
}

\begin{abstract}
RESUMO
Os solos brasileiros, principalmente os do cerrado, são bastante intemperizados e pobres em alguns micronutrientes catiônicos na solução do solo. A utilização de técnicas de manejo, como a adubação verde, pode favorecer o fluxo difusivo (FD) e a disponibilidade desses nutrientes para as plantas. O presente trabalho visou avaliar se a incorporação de adubos verdes ao solo, em diferentes doses e épocas, modifica o FD e a forma iônica de transporte dos micronutrientes $\mathrm{Zn}, \mathrm{Cu}, \mathrm{Fe}$ e $\mathrm{Mn}$ no solo. Para tanto, foram incorporados dois resíduos vegetais largamente cultivados como adubo verde: o feijão guandu (Cajanus cajan) ou o milheto (Pennisetum americanum) por diferentes períodos $(0,15,25,35,45$ e 55 dias) e doses $\left(0,9,18\right.$ e $\left.36 \mathrm{t} \mathrm{ha}^{-1}\right)$ num Latossolo Vermelho, argiloso, em condições de laboratório. Para avaliar o FD, utilizaram-se resinas de troca aniônica (positivamente carregada) e de troca catiônica (negativamente carregada) na forma de lâmina, incubadas junto ao solo em câmaras de difusão durante 15 dias. Os resultados obtidos demonstraram que houve aumento do FD do $\mathrm{Cu}$ e do Fe com o aumento das doses de material vegetal, principalmente no início do período de incubação, e maior fluxo desses dois micronutrientes para a resina aniônica em relação à catiônica, possivelmente por ser o seu transporte no solo mais dependente da formação de complexos organometálicos com carga líquida negativa. Já para $\mathrm{Zn}$ e Mn, o fluxo difusivo foi maior para a resina catiônica. $\mathrm{O}$ aumento do tempo de incubação favoreceu o fluxo difusivo de $\mathrm{Mn}$ e $\mathrm{Zn}$ e reduziu o do $\mathrm{Cu}$ e Fe.
\end{abstract}

Termos de indexação: carbono orgânico, resinas de troca iônica, transporte de íons, complexos organometálicos.

\footnotetext{
${ }^{(1)}$ Recebido para publicação em março de 2005 e aprovado em novembro de 2006.

(2) Doutorando do Programa de Pós-Graduação do em Solos e Nutrição de Plantas da Universidade Federal de Viçosa - UFV. Avenida P.H. Rolfs s/n., CEP 36571-000 Viçosa MG. E-mail: rodinei_pegoraro@yahoo.com.br

(3) Professor do Departamento de Solos, UFV. E-mail: ivosilva@ufv.br

(4) Doutorando do Programa de Pós-Graduação em Solos e Nutrição de Plantas do Departamento de Solos, UFV.

(5) Bolsista do CNPq.
} 


\title{
SUMMARY: DIFFUSIVE FLUX OF CATIONIC MICRONUTRIENTS AS AFFECTED BY TYPE, DOSE AND TIMING OF GREEN MANURE INCORPORATION INTO SOIL
}

\begin{abstract}
Brazilian soils, especially in the Cerrado region, are highly weathered and poor in some cationic micronutrients in the soil solution. Diffusion is the main transport mechanism to the root surface. The adoption of management practices such as the use of green manures might favor the diffusive flux $(D F)$ and availability of micronutrients to plants. The present experiment aimed to evaluate the effect of incorporation of two distinct green manures into a clayey Red Latosol (LV, Typic Haplustox) on the DF of the micronutrients $\mathrm{Zn}, \mathrm{Cu}, \mathrm{Fe}$ and Mn. For this purpose, two widely cultivated green manures: guandu bean (Cajanus cajan) or millet (Pennisetum americanum) were incorporated into the soil for different periods $(0$, $15,25,35,45$, and 55 days) and at variable doses $\left(0,9,18\right.$, and 36 h $^{-1}$, dry matter basis) before starting the evaluation of the DF. To evaluate the $D F$, two ion-exchange membranes (a positively-charged anionic and a negatively-charged cationic exchange resin) were incubated with the soil in diffusion chambers for 15 days. The obtained results demonstrated that there was an increment in the $\mathrm{Cu}$ and $\mathrm{Fe} D \mathrm{~F}$ with the increase in the doses of plant residues, particularly in the beginning of the incubation period. The results also showed a higher DF of these two micronutrients to the anionic resin compared with the cationic resin. This probably occurred because the $\mathrm{Cu}$ and $\mathrm{Fe}$ transport in the soil depends on the formation of organometallic complexes with net negative charge. The DF of Zn and $M n$ to occurred mainly toward the cationic resin. A longer incubation time favored the DF of $\mathrm{Mn}$ and $\mathrm{Zn}$ and reduced that of $\mathrm{Cu}$ and $\mathrm{Fe}$.
\end{abstract}

Index terms: organic carbon, ion-exchange membranes, nutrient transport, organometallic complexes.

\section{INTRODUÇÃO}

Em solos altamente intemperizados, a baixa fertilidade natural requer a adubação corretiva para a maioria dos cultivos comerciais. Nessas condições, os solos podem funcionar muito mais como dreno do que propriamente como fonte de micronutrientes para as plantas. Assim, em adição à fertilização regular, são necessárias práticas de manejo que beneficiem o aporte constante de compostos orgânicos que aumentam a estabilidade das formas mais solúveis de micronutrientes catiônicos na solução do solo. Compostos orgânicos de baixo peso molecular, provenientes de resíduos de plantas e da atividade microbiana no solo, como o citrato e o malato, dentre outros, podem complexar micronutrientes catiônicos ( $\mathrm{Zn}, \mathrm{Cu}, \mathrm{Fe}$ e Mn) do solo e favorecer o seu fluxo difusivo (FD) para a superfície das raízes (Elgawhary et al., 1970; Jones \& Darrah, 1994; Barber, 1995).

Várias práticas agrícolas que visam ao aumento da produtividade e sustentabilidade da produção estão sendo adotadas com maior freqüência, nos últimos anos, no Brasil. Uma delas é a adubação verde que, aliada a sistemas conservacionistas, como o plantio direto, tem proporcionado maior aporte de resíduos vegetais e, conseqüentemente, aumento no conteúdo de matéria orgânica dos solos (Rheinheimer et al., 1998; Alcântara et al., 2000).
Dentre os efeitos da adubação verde sobre as características químicas do solo, destacam-se: o aumento do teor de matéria orgânica, a maior disponibilidade de nutrientes, a maior capacidade de troca de cátions efetiva do solo, além do favorecimento da produção de ácidos orgânicos, os quais são de fundamental importância para a solubilização de minerais e a diminuição do teor de $\mathrm{Al}$ trocável, pela sua complexação (Franchini et al., 2003; Jones, 1998; Alcântara et al., 2000).

Vários compostos orgânicos interagem com os micronutrientes no solo, principalmente naqueles bastante intemperizados, onde predominam óxidos de $\mathrm{Fe}$ e Al com cargas variáveis. Eles podem ligar-se aos sítios de troca dos óxidos, diminuindo-lhes a possibilidade de formarem ligações de caráter covalente com os micronutrientes catiônicos (Stevenson, 1994). A liberação de compostos orgânicos pela mineralização de resíduos vegetais também colabora para a formação de complexos estáveis com os micronutrientes na solução do solo, podendo aumentar sua mobilidade e disponibilidade para as plantas (Silva \& Pasqual, 1999; Miyazawa et al., 2000). Esses efeitos dos compostos orgânicos são atribuídos aos fenômenos de dissolução e complexação de minerais (Ochs, 1996; Hue et al., 2001; Temminghoff et al., 1997), por alterar o $\mathrm{pH}$ do solo (Franchini et al., 1999) e por favorecer a formação de ligações com a superfície dos colóides do solo (Jones, 1998). 
Grande proporção dos micronutrientes $\mathrm{Zn}, \mathrm{Cu}, \mathrm{Fe}$ e Mn na solução do solo encontra-se ligada a compostos orgânicos (Hodgson et al., 1966; Harter \& Naidu 1995), e a difusão é o principal mecanismo de transporte desses para a superfície das raízes das plantas. No entanto, pouco se conhece dos efeitos dos resíduos vegetais no FD de micronutrientes catiônicos e em que forma iônica eles são transportados.

O objetivo do estudo foi avaliar se a incorporação de dois adubos verdes ao solo, em diferentes doses e épocas, modifica o FD e a forma iônica de transporte dos micronutrientes $\mathrm{Zn}, \mathrm{Cu}$, Fe e $\mathrm{Mn}$ no solo.

\section{MATERIAL E MÉTODOS}

O experimento constou de um fatorial $2 \times 4 \times 6$, correspondendo, respectivamente, a dois materiais vegetais incubados com solo em quatro doses e seis períodos de tempo, com quatro repetições, num delineamento inteiramente ao acaso, totalizando 192 unidades experimentais.

Neste experimento, foi utilizado um Latossolo Vermelho (LV) de textura argilosa, do município de São Sebastião do Paraiso, MG. O solo foi coletado a uma profundidade de 0-20 cm, seco ao ar, destorroado, passado em peneira de malha de $2 \mathrm{~mm}$ e homogeneizado. Retiraram-se subamostras para a caracterização química e física (Quadro 1).

O solo recebeu carbonatos de cálcio e de magnésio p.a. (relação molar 4:1), em quantidade suficiente para elevar a saturação por bases a 60 \%. Em seguida, a umidade do solo foi ajustada para a capacidade de campo (potencial $=-30 \mathrm{kPa}$ ). Após 15 dias de incubação em sacos de polietileno, para elevar o pH do solo, o solo foi seco e novamente peneirado (2 $\mathrm{mm})$, com o intuito de favorecer sua homogeneização, para dar início à incorporação dos resíduos vegetais.

\section{Quadro 1. Características físicas e químicas do Latossolo Vermelho (LV), argiloso}

\begin{tabular}{|c|c|}
\hline Característica & $\mathbf{L V}$ \\
\hline Umidade $-30 \mathrm{kPa}\left(\mathrm{kg} \mathrm{kg}^{-1}\right)^{(1)}$ & 0,223 \\
\hline Areia Grossa $(\%)^{(1)}$ & 6,0 \\
\hline Areia Fina $(\%)^{(1)}$ & 24,0 \\
\hline Argila $(\%)^{(1)}$ & 47,0 \\
\hline Silte $(\%)^{(1)}$ & 23,0 \\
\hline $\mathrm{pH}\left(\mathrm{H}_{2} \mathrm{O}\right)$ & 5,06 \\
\hline Carbono orgânico $\left(\mathrm{g} \mathrm{kg}^{-1}\right)^{(2)}$ & 14,90 \\
\hline $\mathrm{Zn}\left(\mathrm{mg} \mathrm{dm}^{-3}\right)^{(3)}$ & 16,59 \\
\hline Fe $\left(\mathrm{mg} \mathrm{dm}^{-3}\right)^{(3)}$ & 54,33 \\
\hline $\operatorname{Mn}\left(\mathrm{mg} \mathrm{dm}^{-3}\right)^{(3)}$ & 55,23 \\
\hline $\mathrm{Cu}\left(\mathrm{mg} \mathrm{dm}^{-3}\right)^{(3)}$ & 10,42 \\
\hline
\end{tabular}

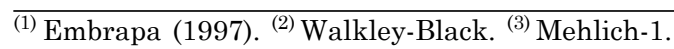

Utilizaram-se dois resíduos vegetais distintos e largamente cultivados como adubo verde: o feijão guandu (Cajanus cajan) e o milheto (Pennisetum americanum). Os materiais vegetais foram coletados em pleno crescimento vegetativo, secos em estufa a $50{ }^{\circ} \mathrm{C}$, por $72 \mathrm{~h}$, moídos em moinho com peneira de $0,5 \mathrm{~mm}$ e então incorporados ao solo por diferentes períodos $(0,15,25,35,45,55$ dias), antes de se avaliar o fluxo difusivo (FD), nas doses equivalentes a $0,9,18$ e $36 \mathrm{t}^{\mathrm{a}} \mathrm{a}^{-1}$ de matéria seca. O primeiro tratamento a receber os materiais vegetais foi o de 55 dias. Dez dias mais tarde, o solo com o tratamento de 45 dias recebeu os adubos verdes e, assim, sucessivamente, de forma que todos os tratamentos tinham sido aplicados por ocasião do início do experimento. $\mathrm{O}$ solo foi incubado com os materiais vegetais em sacos de polietileno, os quais foram vedados, para que a umidade fosse mantida constante, e, semanalmente, esses foram abertos e revolvidos, para que o material vegetal não fosse decomposto sob condições anaeróbias.

Os micronutrientes $\mathrm{Zn}$, Cu, Fe e Mn foram adicionados ao solo 15 dias antes da avaliação do fluxo difusivo, na dose de $10 \mathrm{mg} \mathrm{dm}^{-3}$, utilizando solução com sais de sulfato. Os outros nutrientes foram aplicados em quantidades sugeridas para a realização de estudos com plantas em casa de vegetação (Novais et al., 1991), visando simular as condições ideais de crescimento.

\section{Montagem da câmara de difusão e avaliação do fluxo difusivo}

As câmaras de difusão foram construídas com tubos de PVC com $10 \mathrm{~cm}$ de diâmetro e $5 \mathrm{~cm}$ de altura, vedadas na parte inferior com uma placa de isopor, proporcionando volume de $0,393 \mathrm{dm}^{3}$. Para a montagem da câmara, foi adicionada metade do solo com os respectivos tratamentos e, em seguida, foram acomodadas as lâminas de resina aniônica e catiônica $\left(2,5 \times 4 \mathrm{~cm} ; 10 \mathrm{~cm}^{2}\right.$ por face), e sobre estas o restante do solo. A utilização da resina trocadora de cátions (CR 61 CZR, Ionics Inc., E.U.A.) e da resina trocadora de ânions (204U2ARA, Ionics Inc., E.U.A.), negativamente e positivamente carregadas, respectivamente, teve o objetivo de avaliar o FD de $\mathrm{Zn}, \mathrm{Fe}, \mathrm{Cu}$ e $\mathrm{Mn}$, pois essas serviram de dreno para esses micronutrientes e, portanto, simularam a presença de uma raiz para onde poderiam ser transportados e adsorvidos, da mesma forma que fundamentados por Villani et al. (1998); Oliveira et al. (1999) e Nunes et al. (2004). Cada resina media $2,5 \times 4 \mathrm{~cm}$, totalizando uma superfície de adsorção de $20 \mathrm{~cm}^{2}$, quando consideradas as duas faces da lâmina.

O solo com os tratamentos foi mantido em umidade correspondente à capacidade de campo $(-30 \mathrm{kPa})$ por meio do envolvimento da câmara de difusão com filme de PVC, evitando, assim, a perda de umidade durante o período de determinação do FD. As câmaras foram mantidas em laboratório com uma temperatura ambiente de $25 \pm 2{ }^{\circ} \mathrm{C}$. Decorridos 15 dias, as lâminas 
de cada câmara de difusão foram retiradas, lavadas com água deionizada corrente, para retirar as partículas maiores de solo aderidas, e então agitadas em frascos plásticos, durante $30 \mathrm{~min}$, com água ultrapura (Milli-Q), para retirada de possíveis partículas de solo mais difíceis de ser removidas por água corrente. Em seguida, as lâminas foram colocadas em $\mathrm{HCl} 0,5 \mathrm{~mol} \mathrm{~L}^{-1}$, por $1,5 \mathrm{~h}$, agitadas (150 rpm) para dessorção dos elementos. Nesses extratos, foram determinados os teores de $\mathrm{Zn}, \mathrm{Fe}, \mathrm{Cu}$ e Mn por espectrofotometria de absorção atômica.

\section{Determinação do pH}

Coletaram-se $10 \mathrm{~cm}^{3}$ de solo em potes plásticos de $50 \mathrm{~mL}$, em que foram adicionados $25 \mathrm{~mL}$ de água deionizada. As amostras foram agitadas continuamente, durante $5 \mathrm{~min}$, deixadas em repouso, durante $30 \mathrm{~min}$, e imediatamente mediu-se o $\mathrm{pH}$ por meio de um potenciômetro.

\section{Extração e determinação dos ácidos orgânicos dos resíduos vegetais}

Após o material vegetal ter sido seco e triturado em moinho equipado com peneira de $0,5 \mathrm{~mm}$, pesouse $0,1000 \mathrm{~g}$ do material para maceração em almofariz na presença de $\mathrm{N}$ líquido, com o intuito de favorecer a quebra do tecido vegetal. Posteriormente, o material vegetal foi macerado em $4 \mathrm{~mL}$ de água ultrapura e centrifugado (3.000 g), durante 5 min. Essa operação foi repetida quatro vezes, até que o sobrenadante não mais apresentasse coloração esverdeada. O sobrenadante foi concentrado sob vácuo e, posteriormente, as amostras foram reconstituídas em $1 \mathrm{~mL}$ de água ultrapura e passadas em filtro de membrana de 0,45 $\mu \mathrm{m}$. Antes de proceder-se à análise por cromatografia de íons (Silva et al., 2001), as amostras de milheto foram diluídas novamente 20 vezes e as de feijão guandu 10 vezes.

\section{Análise estatística}

Os resultados foram submetidos à análise de variância, utilizando-se o programa estatístico SAEG (Sistema de Análises Estatísticas e Genéticas) (FUNARBE, 1993) e, quando pertinente, foi realizada análise de regressão.

\section{RESULTADOS}

\section{Fluxo difusivo}

O fluxo difusivo (FD) de $\mathrm{Cu}$ e $\mathrm{Fe}$ foi maior para a resina aniônica e o de $\mathrm{Zn}$ e $\mathrm{Mn}$ para a resina catiônica (Quadro 2). Isso evidencia a possível formação e transporte por difusão de complexos organometálicos com carga líquida negativa, especialmente para os dois primeiros nutrientes. $\mathrm{O}$ milheto foi o resíduo que possibilitou maior FD de $\mathrm{Zn}$, $\mathrm{Cu}$ e $\mathrm{Fe}$ para a resina aniônica (positivamente carregada). Já para a resina cationnica (negativamente carregada), o guandu foi o resíduo que manteve maior FD de $\mathrm{Zn}$ e Mn. O FD do $\mathrm{Cu}$ e Fe para a resina catiônica foi abaixo do limite de detecção, o que evidencia a alta capacidade desse solo em reter esses micronutrientes, e, ou, a baixa solubilidade dos minerais que contêm $\mathrm{Cu}$ e $\mathrm{Fe}$. Isso restringe o seu transporte principalmente a formas orgânicas complexadas, por terem maior solubilidade e mobilidade no solo. O fluxo de Mn para a resina aniônica também foi abaixo do limite de detecção.

O FD do $\mathrm{Zn}$ para a resina aniônica teve comportamento cúbico de acordo com o tempo e foi superior no solo que recebeu resíduo vegetal em relação ao tratamento que não recebeu o resíduo vegetal (Figura 1). As doses de 18 e $36 \mathrm{t} \mathrm{ha}^{-1}$ de feijão guandu promoveram, após 55 dias de incubação, o maior FD de Zn no solo. Verificou-se também que o FD de Zn para a resina catiônica foi beneficiado pelo aumento

Quadro 2. Fluxo difusivo de Zn, Cu, Fe e Mn para a resina aniônica (FDRA) ou a resina catiônica (FDRC) em Latossolo Vermelho argiloso após a incorporação dos resíduos vegetais de guandu ou milheto (valores médios de quatro doses e seis tempos de incubação)

\begin{tabular}{|c|c|c|c|c|c|c|c|c|}
\hline \multirow{2}{*}{$\mathbf{R V}$} & \multicolumn{2}{|c|}{$\mathrm{Zn}$} & \multicolumn{2}{|c|}{$\mathrm{Cu}$} & \multicolumn{2}{|c|}{$\mathbf{F e}$} & \multicolumn{2}{|c|}{ Mn } \\
\hline & FDRA $^{(1)}$ & FDRC (2) & DFRA & DFRC & DFRA & DFRC & DFRA & DFRC \\
\hline Guandu & $0,39 \mathrm{~b}^{(3)}$ & $9,23 \mathrm{a}$ & $1,63 \mathrm{~b}$ & ND & $30,50 \mathrm{~b}$ & ND & ND & $662,31 \mathrm{a}$ \\
\hline Milheto & $0,59 \mathrm{a}$ & $5,46 \mathrm{~b}$ & $3,36 \mathrm{a}$ & ND & $50,39 \mathrm{a}$ & ND & ND & $612,03 \mathrm{~b}$ \\
\hline Média & 0,49 & 7,35 & 2,50 & ND & 40,44 & ND & ND & 637,17 \\
\hline
\end{tabular}

(1) Resina aniônica (carregada positivamente). ${ }^{(2)}$ Resina catiônica (carregada negativamente). (3) Valores médios seguidos pela mesma letra, na coluna, não diferem significativamente pelo teste $\mathrm{F}(\mathrm{P}<0,001)$. ND: abaixo do limite de detecção. 
do tempo de incubação dos materiais vegetais. No entanto, o FD aos 55 dias foi favorecido pelas menores doses de material vegetal (Figura 1).

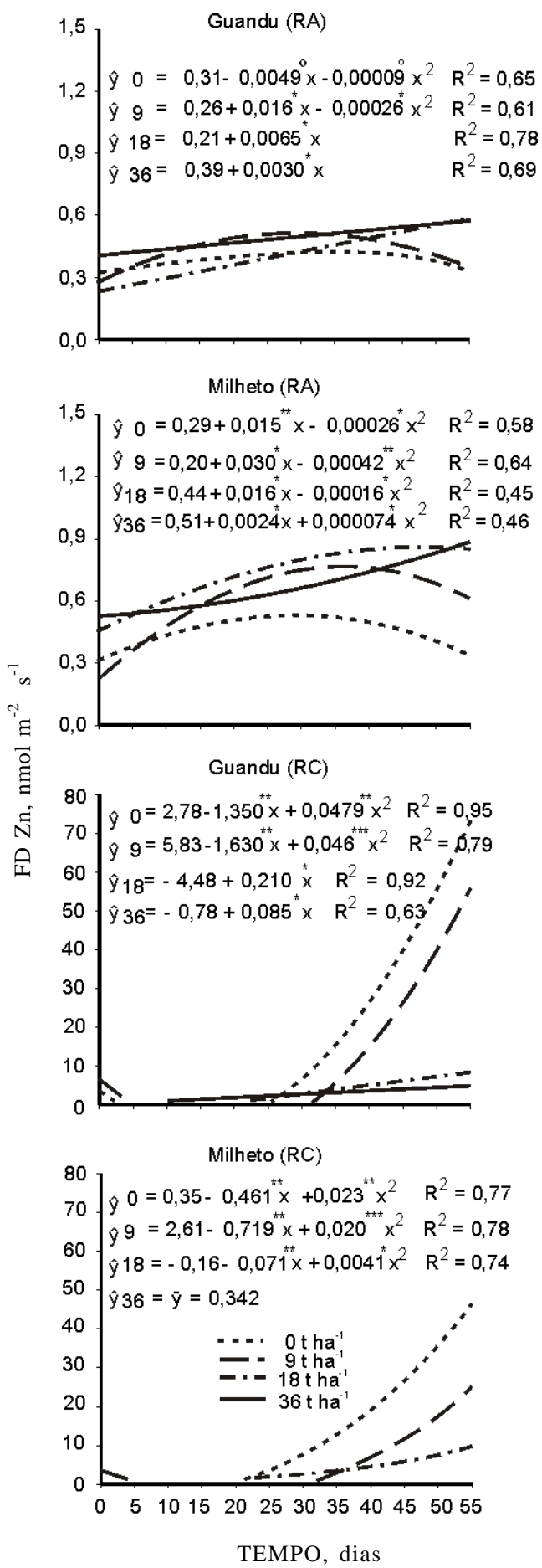

Figura 1. Fluxo difusivo de $\mathrm{Zn}$ para as resinas: aniônica (RA) e catiônica (RC), em Latossolo Vermelho argiloso influenciado pelo tempo de incubação e doses de feijão guandu e milheto. ${ }^{\text {ns }}$, ${ }^{\circ},,^{* * *},{ }^{* * * *}$ : não-significativo e significativos a 10, 5, 1 e $0,1 \%$, respectivamente.
O FD de Cu para a resina aniônica, com ambos os resíduos vegetais, apresentou comportamento semelhante ao longo do tempo de incubação, embora o valor inicial encontrado para a dose de $36 \mathrm{t} \mathrm{ha}^{-1} \mathrm{de}$ milheto tenha sido duas vezes maior que os maiores valores de $\mathrm{FD}$ de $\mathrm{Cu}$ no solo que recebeu guandu (Figura 2). O FD do $\mathrm{Cu}$ foi maior para os períodos de incubação mais curtos, e, após 55 dias de incubação, obtiveram-se valores bastante baixos para todos os tratamentos testados.

A adição de doses crescentes de adubos verdes ao solo aumentou o FD de Fe para a resina aniônica, e com o aumento do tempo de incubação até 25 dias, na maioria das doses estudadas, posteriormente a este período, ocorreu redução do FD até os 55 dias (Figura 2). O Mn foi o elemento que apresentou maior FD para a resina catiônica, tendo-se observado acentuado aumento na sua difusão, quando houve maior tempo de incubação e na presença de maiores doses de guandu e milheto.

A maior liberação de compostos hidrossolúveis pela adição de resíduos vegetais é, provavelmente, a principal razão para esse comportamento. Deve-se considerar também a adição de nutrientes via resíduos vegetais como fator que pode contribuir para o aumento do FD dos nutrientes ao solo, mesmo que esta contribuição seja bem inferior à dose de $10 \mathrm{mg} \mathrm{dm}^{-3}$ dos quatro micronutrientes adicionada ao solo (Quadro 3).

Foram determinados por cromatografia iônica os diversos ácidos orgânicos presentes nos materiais vegetais, cabendo ressaltar que, anteriormente à determinação, as amostras de milheto foram diluídas em água 20 vezes e as de feijão guandu 10 vezes (Figura 3). Encontrou-se maior concentração de ânions orgânicos, especialmente malato e oxalato, no extrato do milheto do que no material vegetal do feijão guandu (Quadro 3). Esse resultado pode explicar a maior eficiência do milheto em manter maior FD de alguns micronutrientes catiônicos no solo, tais como o $\mathrm{Cu}$ e o $\mathrm{Fe}$, principalmente para a resina aniônica.

\section{pH do solo}

A adição de material vegetal ao solo também proporcionou alteração no seu $\mathrm{pH}$. De modo geral, seu efeito foi mais pronunciado nos primeiros 15 dias de incubação, tanto para guandu quanto para milheto. Do tempo 0 aos 55 dias de incubação, ocorreu mudança de natureza quadrática para todos os tratamentos, tendendo a decréscimo em relação ao tempo de incubação (Figura 4). Contudo, essa redução parece ter sido atenuada pelas doses crescentes de material vegetal, principalmente do milheto, possivelmente pela maior presença de ânions orgânicos em comparação ao guandu.

\section{DISCUSSÃO}

A determinação do FD de nutrientes contempla uma região específica do solo onde as reações de dissolução, complexação ou adsorção entre o nutriente, 

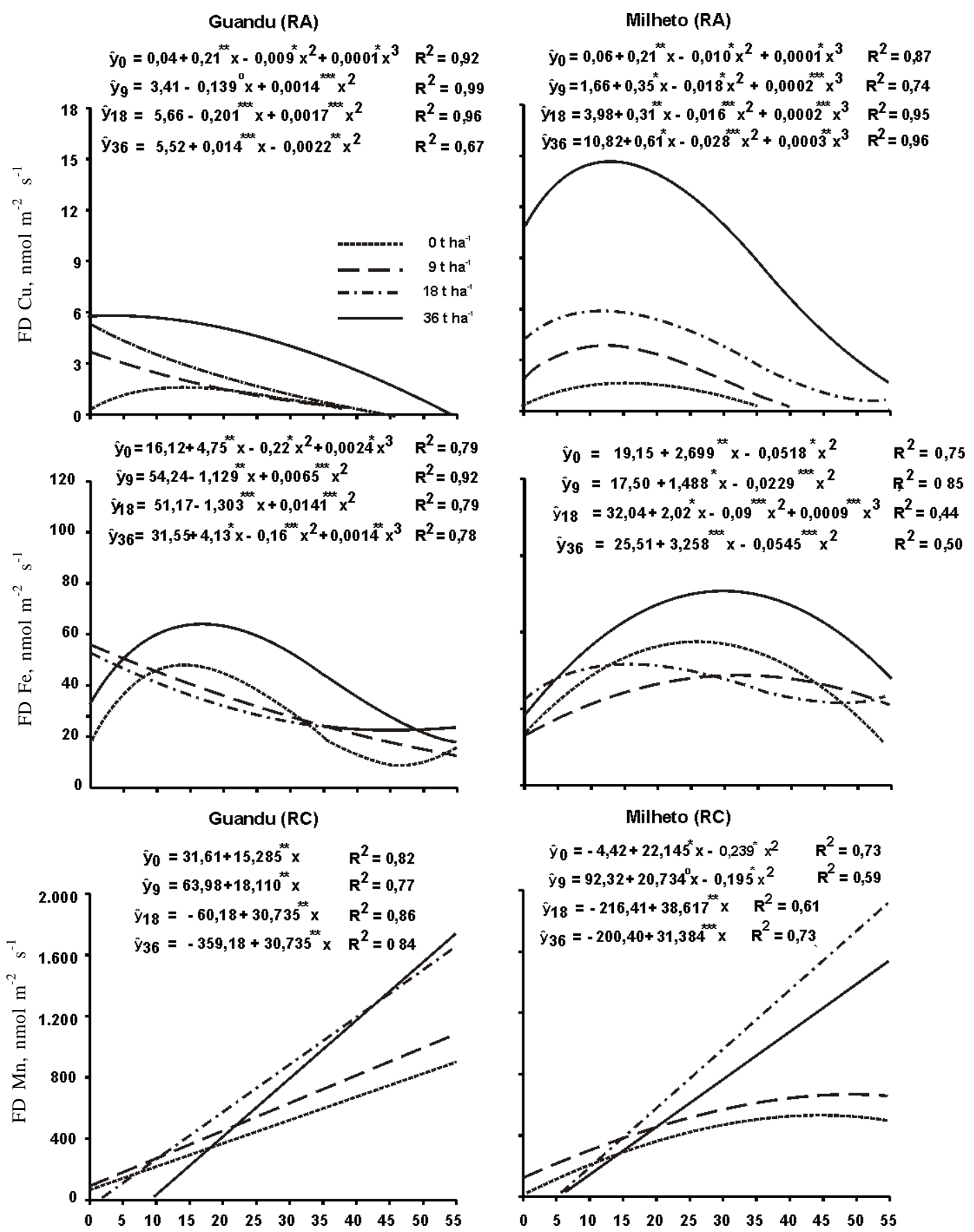

Milheto (RC)

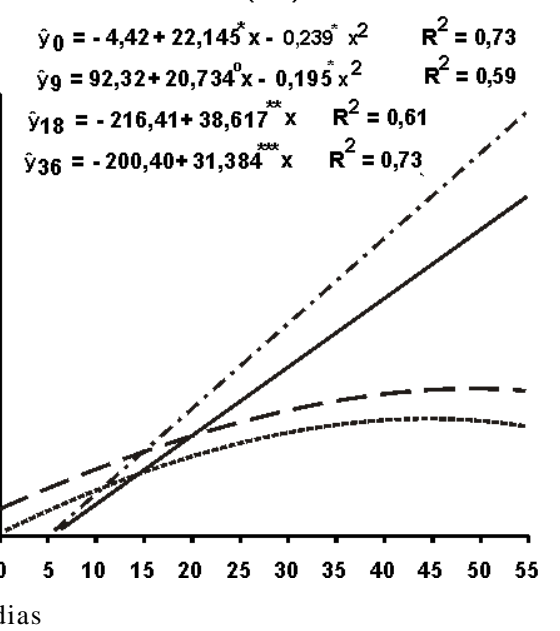

Figura 2. Fluxo difusivo de Cu e Fe para as resinas: aniônica (RA) e de Mn para a resina catiônica (RC) em Latossolo Vermelho argiloso influenciado pelo tempo de incubação e doses de feijão guandu e milheto.

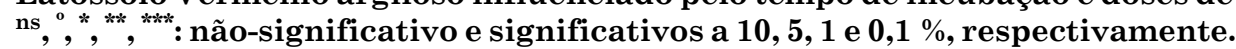

os colóides inorgânicos e os compostos orgânicos ocorrem na presença de um "simulador radicular" (Barber, 1995). As resinas de troca iônica podem ser usadas com tal finalidade, uma vez que apresentam capacidade de adsorver o nutriente ou o composto transportado por diferença de concentração até à superfície da lâmina ionicamente carregada, durante determinado período de tempo. Dessa forma, o processo está sujeito, da mesma forma que a raiz da planta, à competição com a superfície dos argilominerais, microrganismos e matéria orgânica do solo pelo nutriente, o que proporciona melhor representação temporal do que pode ser transportado a curtas distâncias (Pegoraro et al., 2005). 
Quadro 3. Teores dos ácidos orgânicos determinados por cromatografia de íons nos resíduos vegetais de guandu e milheto

\begin{tabular}{|c|c|c|c|c|c|c|c|c|c|c|}
\hline \multirow{2}{*}{$\begin{array}{l}\text { Resíduo } \\
\text { vegetal }\end{array}$} & \multicolumn{10}{|c|}{ Ácido orgânico } \\
\hline & Acetato & Formato & Butarato & Succinato & Malato & Malonato & Fumarato & Oxalato & Citrato & Isocitrato \\
\hline Guandu & 126,9 & 217,9 & 1,5 & ND & 37,7 & 14,7 & 15,9 & 23,3 & 24,9 & ND \\
\hline Milheto & 73,0 & 312,9 & 46,8 & 72,7 & 502,2 & ND & 36,2 & 137,8 & 27,6 & 34,4 \\
\hline
\end{tabular}

ND: abaixo do limite de detecção.

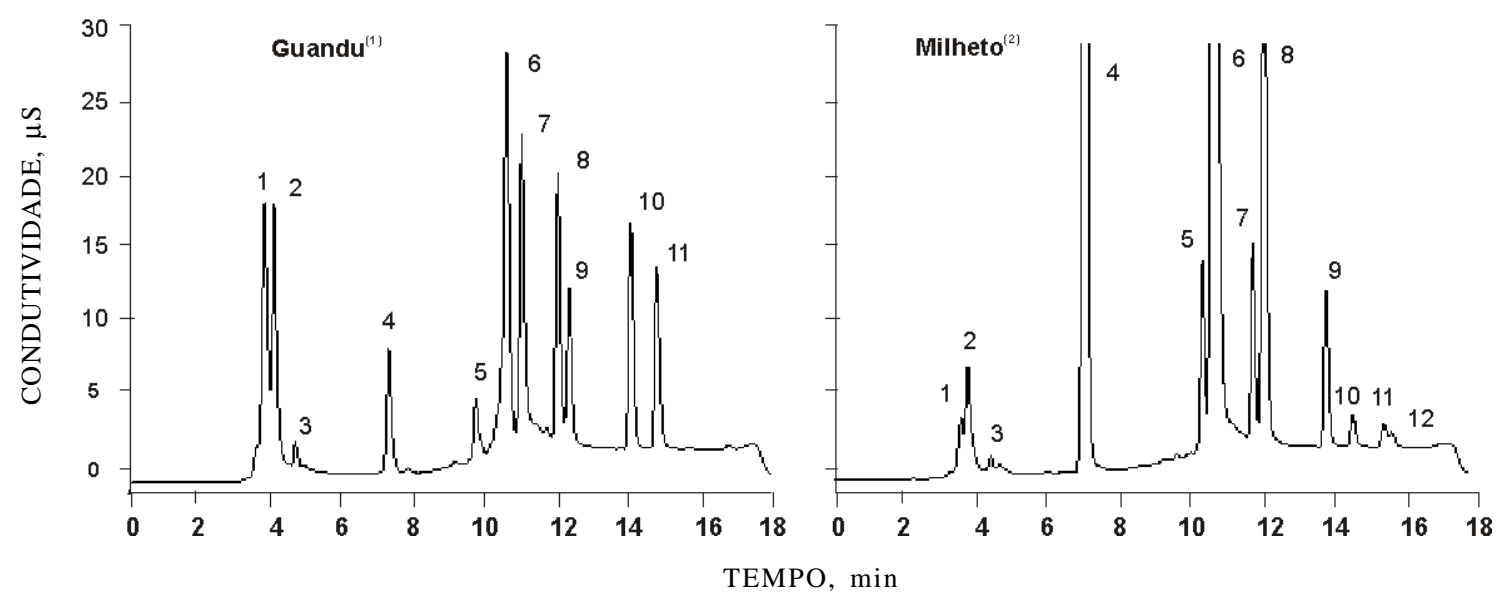

Figura 3. Cromatogramas demonstrando a presença de ácidos orgânicos e ânions inorgânicos nos extratos vegetais de guandu e milheto. Guandu: 1 . acetato, 2. formato, 3. butarato, 4. cloreto, 6. malato, 7. malonato, 8. fumarato, 9. oxalato, 10. fosfato, 11. citrato; Milheto: 1. acetato, 2 . formato, 3. butarato, 4. cloreto, 5. succinato, 6. malato, 7. fumarato, 8. oxalato, 9. fosfato, 10. citrato, 12. isocitrato. ${ }^{(1)}$ Fator de diluição: 10 vezes; ${ }^{(2)}$ Fator de diluição: 20 vezes.
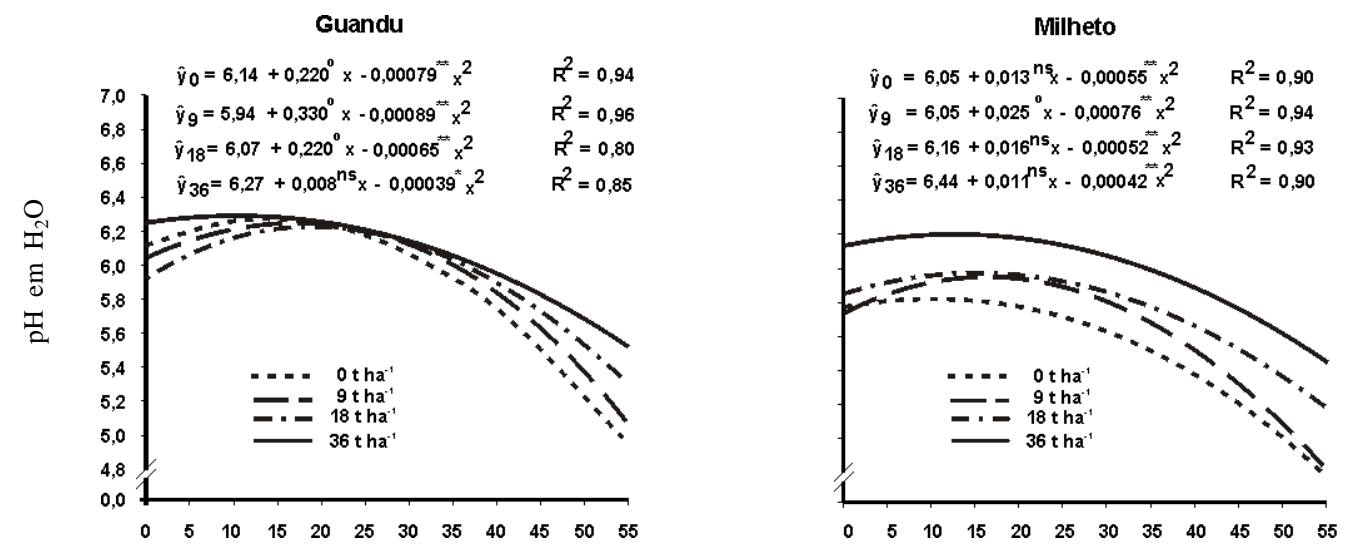

TEMPO, dias

Figura 4. pH do Latossolo Vermelho argiloso afetado pelo tempo de incubação e doses crescentes de feijão guandu e milheto. ${ }^{\mathrm{ns}},{ }^{\circ}, *,{ }^{* * *},{ }^{* * * *}$ : não-significativo e significativos a 10, 5, 1 e 0,1 \%, respectivamente.

De acordo com os resultados, a adição de resíduos vegetais ao solo aumenta o FD de $\mathrm{Zn}$, Cu e Fe para a resina aniônica (Figuras 1 e 2), possivelmente como complexos organometálicos. A liberação de ácidos orgânicos do tecido vegetal (Figura 4) favorece a formação desses complexos com os íons metálicos do 
solo e contribui para a elevação do pH e CTC do solo (Franchini et al., 1999); o primeiro, principalmente, pela reação de troca de ligantes entre ânions orgânicos e o $\mathrm{OH}$ terminal em óxidos de $\mathrm{Fe}$ e $\mathrm{Al}$ (Hue \& Amien, 1989) e o segundo, pelo aumento da saturação por bases no complexo coloidal do solo resultante do aumento de cargas dependentes de $\mathrm{pH}$. Temminghoff et al. (1997) observaram que, no solo com pH 6,6, mais de $99 \%$ do $\mathrm{Cu}$ em solução estava complexado por compostos orgânicos de baixo peso molecular. Além disso, a presença simultânea de ácidos orgânicos e desses metais na resina aniônica e não na catiônica (Pegoraro, 2003) constitui forte evidência para tal hipótese. Portanto, a presença de ligantes orgânicos no solo parece ter grande importância para a manutenção de formas disponíveis de alguns micronutrientes.

Os micronutrientes catiônicos apresentam diferentes graus de afinidade com os compostos orgânicos do solo, e essa afinidade geralmente segue a ordem descrita por Irwing-Williams: $\mathrm{Fe}^{3+}>\mathrm{Cu}^{2+}>$ $\mathrm{Zn}^{2+}>\mathrm{Fe}^{2+}>\mathrm{Mn}^{2+}$. Essa seqüência nem sempre é observada, pois existem vários fatores, tais como: a natureza do sítio de carga negativa e sua conformação molecular, que podem alterar a afinidade de um ligante pelo cátion (Canellas et al., 1999). Os dados de FD para $\mathrm{Fe}$ e $\mathrm{Cu}$ mostraram que existe maior afinidade desses com os compostos orgânicos em comparação ao $\mathrm{Zn}$ e $\mathrm{Mn}$, ou seja, uma fração maior dos micronutrientes foi transportada para a resina aniônica, possivelmente, pela formação de complexos organometálicos com carga líquida negativa. Já o Mn difundiu quase que exclusivamente para a resina catiônica (negativamente carregada) e o Zn apresentou comportamento intermediário. Agbenin et al. (1999), trabalhando na extração de $\mathrm{Fe}, \mathrm{Cu}, \mathrm{Zn}$ e $\mathrm{Mn}$ com resina de troca iônica mista modificada, saturada com ligantes orgânicos e inorgânicos, observaram que a resina saturada com citrato de sódio $0,5 \mathrm{~mol} \mathrm{~L}^{-1}$ extraiu mais $\mathrm{Cu}$ que outras resina-ligantes testadas ( NaCl 0,5 mol L-1; $\mathrm{NaHCO}_{3} 0,5 \mathrm{~mol} \mathrm{~L}^{-1} ; \mathrm{NaF}$ $0,5 \mathrm{~mol} \mathrm{~L}^{-1}$; NaOAc $0,5 \mathrm{~mol} \mathrm{~L}^{-1} \mathrm{e}$ tartarato de sódio $0,5 \mathrm{~mol} \mathrm{~L}^{-1}$ ). Os resultados encontrados no presente estudo corroborou os encontrados por esses mesmos autores que também reportaram que a solubilidade de $\mathrm{Fe}$ e de $\mathrm{Cu}$ é fortemente controlada por complexos orgânicos.

Para $\mathrm{Cu}$ e $\mathrm{Fe}$, verificou-se maior FD no início do período de incubação ( \pm 25 dias), evidenciando que a liberação de compostos orgânicos hidrossolúveis lixiviados do próprio tecido vegetal aplicado contribuiu para a dissolução e formação de complexos com o Fe e $\mathrm{Cu}$ do solo. Os compostos orgânicos, como os ácidos orgânicos de baixo peso molecular (ex.: citrato, malato, etc.), foram identificados nos materiais vegetais utilizados (Figura 3, Quadro 3) e rapidamente liberados quando em contato com água (Carvalho, 2003), podendo formar ligações com os sítios de adsorção dos argilominerais do solo (van Hees et al., 2003), favorecer a dissolução de minerais (Strobel, 2001), além de formar complexos com os micronutrientes na solução do solo e reduzir a possibilidade da precipitação como óxidos de menor solubilidade (Lindsay, 1979; Bayer \& Mielniczuk, 1999). Isso, geralmente, resulta em aumento na sua disponibilidade para as plantas.

Com o passar do tempo, esses compostos lixiviados do material vegetal para a solução do solo são fortemente adsorvidos pelos colóides inorgânicos do solo (Jones, 1998) e, ou decompostos pela atividade microbiana do meio, reduzindo, assim, o potencial de complexação (Franchini et al., 1999; 2001).

De maneira contrastante ao $\mathrm{Fe} \mathrm{e} \mathrm{Cu}$, o FD do $\mathrm{Zn}$ e do Mn aumentou com o tempo de incubação dos resíduos vegetais, e isso pode estar associado à redução do $\mathrm{pH}$ do solo com o aumento do tempo de incubação, possibilitando o aumento do $\mathrm{H}^{+}$em solução do solo que, juntamente da presença de ácidos orgânicos de baixo peso molecular, pode ter favorecido a dissolução e a liberação do $\mathrm{Zn}^{2+}$ e do $\mathrm{Mn}^{2+}$ para a solução do solo. Godo \& Reisenauer (1980) demonstraram o importante papel do malato na dissolução de $\mathrm{MnO}_{2}$ e o aumento do $\mathrm{Mn}^{2+}$ na solução do solo:

$$
\begin{gathered}
\text { Malato }^{2-}{ }_{\text {sol. }}+\mathrm{MnO}_{2 \text { solo }}+2 \mathrm{H}^{+}= \\
\text {oxaloacetato }^{2-}{ }_{\text {sol. }}+\mathrm{Mn}^{2+}{ }_{\text {sol. }}+\mathrm{H}_{2} \mathrm{O}
\end{gathered}
$$

De fato, Hue et al. (2001) verificaram que, ao adicionar 5 e $10 \mathrm{~g} \mathrm{~kg}^{-1}$ de folhas de Vigna unguiculata L. e lodo de esgoto em solos do Havaí ricos em Mn, houve incremento da concentração de $\mathrm{Mn}$ na solução do solo e na parte aérea da cultura da soja (chegando a atingir teores fitotóxicos) devido ao maior efeito de dissolução e de complexação desse nutriente no solo, demonstrando que a adição de compostos orgânicos ao solo cria um ambiente de redução, diminuindo o suprimento de $\mathrm{O}_{2}$ devido à intensa atividade microbiana e produzindo compostos orgânicos que podem dissolver e manter alta concentração de Mn em solução (Hue et al., 2001).

A adição de resíduo vegetal ao solo também proporcionou aumento no $\mathrm{pH}$, principalmente nos primeiros 15 dias de incubação, e foi mais acentuada para os tratamentos com milheto. Franchini et al. (1999) observaram que, após a aplicação de extratos de resíduos de adubos verdes (aveia e nabo), houve aumento significativo do $\mathrm{pH}$ do solo. Esse efeito está de acordo com resultados de pesquisa sob condições de campo, onde a manutenção de material vegetal na superfície do solo em sistemas plantio direto estabelecidos há oito anos proporcionou a manutenção do $\mathrm{pH}$ em água de 0,4 a 0,5 unidade superior àquele no sistema convencional (Rheinheimer et al., 1998).

A elevação do $\mathrm{pH}$ do solo pode ser atribuída não só à adsorção do $\mathrm{H}^{+}$pelos grupos funcionais de superfície dos compostos orgânicos dos resíduos vegetais, que são liberados, em maior quantidade, no início da decomposição do material vegetal (Amaral et al., 2000), mas também à adsorção e precipitação de $\mathrm{Al}^{3+}$ (Miyazawa et al., 1993). O deslocamento de $\mathrm{OH}^{-}$da 
superfície dos colóides atribuído à troca de ligantes com os grupamentos carboxílicos dos ácidos orgânicos também é outra possibilidade. No entanto, essas reações da fração hidrossolúvel dos resíduos vegetais com o solo são muito rápidas e declinam drasticamente com o passar do tempo (Franchini et al., 1999; 2001), o que também foi verificado no presente estudo, após 15 dias de incubação, com a redução de uma unidade do $\mathrm{pH}$ do solo até o período de 55 dias.

Os resultados do presente estudo reforçam a importância da utilização de práticas de manejo que levem a um aporte contínuo de material vegetal ao solo e, conseqüentemente, de compostos orgânicos que mantenham ao longo do tempo quantidades suficientemente móveis desses micronutrientes e que possam ser transportados para as raízes, mantendo o bom desenvolvimento das plantas. Nesse contexto, espécies ou genótipos que tenham maior capacidade de secretar compostos orgânicos, como, por exemplo, ácidos orgânicos na rizosfera apresentam maior capacidade de adaptação a solos deficientes em determinados micronutrientes (Rengel, 2002), o que passa pelo aumento do FD de formas complexadas.

Finalmente, deve-se ressaltar que as evidências apresentadas suportando o FD de complexos organometálicos são indiretas e esses possíveis complexos devem ser isolados e caracterizados em maior detalhe em estudos futuros.

\section{CONCLUSÕES}

1. A presença de resíduos vegetais aumenta o FD de $\mathrm{Zn}, \mathrm{Cu}, \mathrm{Fe}$, como espécies aniônicas, aparentemente como complexos organometálicos com carga líquida negativa, e de Mn, como espécie catiônica.

2. O maior tempo de incubação do material vegetal no solo reduz o FD de $\mathrm{Cu}$ e $\mathrm{Fe}$, para a resina aniônica, e aumenta aquele do $\mathrm{Mn}$ e Zn, para a resina catiônica.

3. De modo geral, o milheto mostrou-se mais eficiente em aumentar o FD dos micronutrientes que o guandu.

\section{AGRADECIMENTOS}

Os autores são gratos a dois revisores anônimos pelas valiosas sugestões apresentadas, especialmente ao "revisor C".

O Prof. Ivo R. Silva agradece o suporte financeiro da FAPEMIG - Fundação de Amparo a Pesquisa do Estado de Minas Gerais por meio do Convênio CAG $882 / 04$.

\section{LITERATURA CITADA}

AGBENIN, J.O.; ABREU, C.A. \& RAIJ, B. van. Extraction of phytoavailable trace metals from tropical soils by mixed ion exchange resin modified with inorganic and organic ligands. Sci. Total Environ., 227:187-196, 1999.

ALCÂNTARA, F.A.; FURTINI NETO, A.E.; PAULA, M.B.; MESQUITA, H.A. \& MUNIZ, J.A. Adubação verde na recuperação da fertilidade de um Latossolo VermelhoEscuro degradado. Pesq. Agropec. Bras., 35:277-288, 2000.

AMARAL, A.S.; SPADER, V.; ANGHINONI, I. \& MEURER, E.J. Resíduos vegetais na superfície do solo afetam a acidez do solo e a eficiência do herbicida flumetsulam. Ci. Rural, 30:789-794, 2000.

BARBER, S.A. Soil nutrient bioavailability: A mechanistic approach. 2.ed. New York, John Wiley \& Sons, 1995. 414p.

BAYER, C. \& MIELNICZUK, J. Dinâmica e função da matéria orgânica. In: SANTOS, G.A. \& CAMARGO, F.A.O., eds. Fundamentos da matéria orgânica do solo: Ecossistemas tropicais \& subtropicais. Porto Alegre, Gênesis, 1999. p.9-23.

CANELLAS, L.P.; SANTOS, G.A. \& AMARAL SOBRINHO, N.M.B. Reações da matéria orgânica. In: SANTOS, G.A. \& CAMARGO, F.A.O., eds. Fundamentos da matéria orgânica do solo: Ecossistemas tropicais e subtropicais. Porto Alegre, Gênesis, 1999. p.69-86.

CARVALHO, G.R. Ácidos orgânicos, açúcares e nutrientes em lixiviados de materiais vegetais. Viçosa, Universidade Federal de Viçosa, 2003. 48p. (Tese de Mestrado)

ELGAWHARY, S.M.; LINDSAY, W.L. \& KEMPER, W.D. Effect of complexing agents and acids on the diffusion of zinc to a simulated root. Soil Sci. Soc. Am. Proc., 34:211-214, 1970.

EMPRESA BRASILEIRA DE PESQUISA AGROPECUÁRIA EMBRAPA. Centro Nacional de Pesquisa de Solos. Manual de métodos de análise de solo. 2.ed. Rio de Janeiro, Ministério da Agricultura e do Abastecimento, 1997. 212p.

FRANCHINI, J.C.; GONZALEZ-VILA, F.J.; CABRERA, F.; MIYAZAWA, M. \& PAVAN, M.A. Rapid transformations of plant water-soluble organic compounds in relation to cation mobilization in an acid Oxisol. Plant Soil, 231:55$63,2001$.

FRANCHINI, J.C.; HOFFMAN-CAMPO, C.B.; TORRES, E.; MIYAZAWA, M. \& PAVAN, M.A. Organic composition of green manure during growth and its effects on cation mobilization in an acid Oxisol. Comm. Soil Sci. Plant Anal., 34:2045-2058, 2003.

FRANCHINI, J.C.; MALAVOLTA, E.; MIYAZAWA, M. \& PAVAN, M.A. Alterações químicas em solos ácidos após a aplicação de resíduos vegetais. R. Bras. Ci. Solo, 23:533$542,1999$.

FUNDAÇÃO ARTHUR BERNARDES - FUNARBE. SAEG Sistema para análise estatística 5.0. Viçosa,MG, 1993.

GODO, G.H. \& REISENAUER, H.M. Plant effects on soil manganese availability. Soil Sci. Soc. Am. J., 44:993-995, 1980. 
HARTER, R.D. \& NAIDU, R. Role of metalorganic complexation in metal sorption by soils. Adv. Agron., 5:219-263, 1995.

HODGSON, J.F.; LINDSAY, W.L. \& TRIERWEILER, J.F. Micronutrient cation complexing in soil solution: II. Complexing of zinc and copper in displaced solution from calcareous soils. Soil Sci. Soc. Am. Proc., 30:723-726, 1966.

HUE, N.V. \& AMIEN, I. Aluminum detoxification with green manures. Comm. Soil. Sci. Plant Anal., 20:1499-1511, 1989.

HUE, N.V.; VEGA, S. \& SILVA, J.M. Manganese toxicity in a Hawaiian Oxisol affected by soil $\mathrm{pH}$ and organic amendments. Soil Sci. Soc. Am. J., 65:153-160, 2001.

JONES, D.L. Organic acids in the rhizosphere - A critical review. Plant Soil, 205:25-44, 1998.

JONES, D.L. \& DARRAH, R. Role of root derived organic acids in mobilization of nutrients from the rhizosphere. Plant Soil, 166:247-257, 1994.

LINDSAY, W. Chemical equilibria in soil. New York, John Wiley \& Sons, 1979. 449p.

MIYAZAWA, M.; PAVAN, M.A. \& CALEGARI, A. Efeito de material vegetal na acidez do solo. R. Bras. Ci. Solo, 17:411-416, 1993.

MIYAZAWA, M.; PAVAN, M.A. \& FRANCHINI. J.C. Resíduos vegetais: influência na química de solos ácidos. In: SIMPÓSIO SOBRE FERTILIDADE DO SOLO E NUTRIÇÃO DE PLANTAS NO SISTEMA PLANTIO DIRETO, 1., Ponta Grossa, 2000. Anais. Ponta Grossa, 2000. p.82-94.

NOVAIS, R.F.; NEVES, J.C.L. \& BARROS, N.F. Ensaio em ambiente controlado. In: OLIVEIRA, A.J.; GARRIDO, W.E.; ARAUJO, J.D. \& LOURENÇO, S. Métodos de pesquisa em fertilidade do solo. Brasília, EMBRAPA-SEA, 1991. p.189-254.

NUNES, F.N.; NOVAIS, R.F.; SILVA, I.R.; GEBRIM, F.O. \& SÃO JOSÉ, J.F.B. Fluxo difusivo de ferro em solos sob influência de doses de fósforo e de níveis de acidez e umidade. R. Bras. Ci. Solo, 28:423-429, 2004.

OCHS, M. Influence of humified and non-humified natural organic compounds on mineral dissolution. Chem. Geol., 132:119-124, 1996.

OLIVEIRA, M.F.G.; NOVAIS, R.F.; NEVES, J.C.L.; ALVES, V.M.C. \& VASCONCELLOS, C.A. Fluxo difusivo de zinco em amostras de solo influenciado por textura, íon acompanhante e pH do solo. R. Bras. Ci. Solo, 23:609-615, 1999.
PEGORARO, R.F. Fluxo difusivo de micronutrientes catiônicos em resposta a adição de compostos orgânicos ao solo. Viçosa, MG, Universidade Federal de Viçosa, 2003. 78p. (Tese de Mestrado)

PEGORARO, R.F.; SILVA, I.R.; NOVAIS, R.F.; MENDONÇA, E.S.; ALVARES V., V.H.; NUNES, F.N.; FONSECA, F.M. \& SMYTH, T.J. Diffusive flux of cátionic micronutrients in two Oxisols as affected by low-molecular-weight organic acid and cover-crop residue. J. Plant Nutr. Soil Sci., 168:334-341, 2005.

RENGEL, Z. Genetic control of root exudation. Plant Soil, 245:59-70, 2002.

RHEINHEIMER, D.S.; KAMINSKI, J.; LUPATINI, G.C. \& SANTOS, E.J.S. Modificações em atributos químicos de solo arenoso sob sistema plantio direto. R. Bras. Ci. Solo, 22:713-721, 1998.

SILVA, I.R.; SMYTH, T.J.; RAPER, C.D.; CARTER, T.E. \& RUFTY, T.W. Differential aluminum tolerance in soybean: An evaluation of the role of organic acids. Physiol. Plant., 112:200-210, 2001.

SILVA, L.M.V. \& PASQUAL, A. Dinâmica e modelagem da matéria orgânica do solo com ênfase ao ecossistema tropical. Energ. Agric., 14:13-24, 1999.

STEVENSON, F.J. Humus chemistry: genesis, composition and reactions. 2.ed. New York, University of Illinois, 1994. 496p.

STROBEL, B.W. Influence of vegetation on low-molecularweight carboxylic acids in soil solution: a review. Geoderma, 99:169-198, 2001.

TEMMINGHOFF, E.J.M.; van der ZEE, S.E.A.T.M. \& DE HAAN, F.A.M. Copper mobility in a copper-contaminated sandy soil as affected by $\mathrm{pH}$ and solid and dissolved organic matter. Environ. Sci. Technol., 31:1109-1115, 1997.

van HEES, P.A.W.; VINOGRADOFF, A.C.; EDWARDS, A.C.; GODBOLD, D.L. \& JONES, D.L. Low molecular weight organic acid adsorption in forest soils: Effects on soil solution concentrations and biodegradation rates. Soil Biol. Biochem., 35:1015-1026, 2003.

VILLANI, E.M.A.; BARROS, N.F.; NOVAIS, R.F.; COMERFORD, N.B.; COSTA, L.M.; NEVES, J.C.L. \& ALVAREZ V., V.H. Phosphorus diffusive flux as affected by phosphate source and incubation time. Soil Sci. Soc. Am. J., 62:1057-1061, 1998. 